\title{
An advanced implementation idea for detecting real-time object occupancy
}

\author{
Claremary James \\ Varghese James
}

\author{
MIDDLE EAST COLLEGE \\ Middle East College
}

\begin{abstract}
Object detection is a title that has earned significances over several fields which have always benefitted socially during circumstances, namely incidents involving human endangerment such as natural disaster where threat may occur in the form of an earthquake, human entrapment underneath rubbles per se. The usage of PIR (Passive Infrared Rays) motion detector to detect humans, objects and other living beings through their movement, has proven the ability in handling situations where such detection is the best chance. However, this approach is not utilized in every situation. In the proposed research paper, an object occupancy detection technology notion is detailed which will describe the function to detect the occupancy or presence of human in an area, specifically transport vehicles that will help in determining passengers inside and to find lost objects as well. The motive behind raising this technological need is to aid or assist in occurrence wherein facing difficulty to find an object being lost or misplaced in a space, as well to detect the humans occupied. This assistance shall ease the detection of occupancy and identifying the lost object. The comprehended object occupancy idea is utilized to recognize the humans and object detection. The implementation idea shall facilitate the utilization of PIR-based motion detector sensor to recognize human presence as well as SlimYOLOv3 framework to identify objects. Circumstances where the occupancy of humans are counted and object to be identified are the main output.
\end{abstract}

\section{Introduction}

The object detection have been a major role when it comes to business sectors which are many in varied aspects such as facial recognition as a biometrics broadly used for authorizing individual based on their facial detection and identification of the person which will act as a device for granting access after ensuring the person is authorized to enter a specific area that requires authorization. Biometrics is a digital object detection technique that evaluates a person's feature which is used in many Companies for personnel authorization to prevent unauthorized person from entering a Company's specific secured area. Once such type is iris code for identification verification which is widely used in many business sectors where authentication is a must. The iris patterns vary among different individuals which will help differentiate each person (Nguyen et al. 2017). This method has increased its popularity in recent years since the technological advancement has been more efficient (Biswas et al. 2017).

Apart from biometrics there are other sectors where the usage of object detection has been tremendously incremented. The frequently used object detection technique was Convolutional Neural Networks (CNN). However, the object detection have led to a great breakthrough in the field of Computer Vision as the latest approach is YOLO (You Only Look Once) which is under Region-based Convolutional Neural Network (R-CNN) where a particular object in an image is located within bounding boxes containing cells once an image is segmented into grids and thereby evaluated using a set of algorithms on dataset namely, Pascal VOC dataset Mirror. The mentioned technique is a prediction-based real-time object detection and therefore can be used vastly in situations where certain objects in an image requires to be easily identifiable in terms of its location in the image as well as its features (Redmon et al. 2016). 


\section{Journal of Student Research}

Fourth Middle East College Student Research Conference, Muscat, Sultanate of Oman

One such example is vehicle license plate detection wherein a vehicle's number plate image can be segmented and determine each character to recognize the license plate. According to machine's view or in other words Computer Vision, can be made possible by having an object detection software with which an object or a person can be recognized in an image by having these objects being extracted. Another sector where object detection is useful is in health sector for medical image-processing, however there is still challenge faced in this field as lack of enough dataset, variation in the balancing of information as well as sophistication for the interpretation of the algorithms which makes it difficult to understand and in overall makes it an incomplete innovation (Razzak et al. 2017). The usages are limitless for detecting of object but some other needful usages of object detection method would be in manufacturing industry where it is required in the assembly line and for maintaining quality management, in the robotics where the robots can be developed to adopt the behavior of human beings as to recognize things around them which gives them the ability where in a real-time scenario for processing the visual data, detecting varied emotion among humans, automated systems for annotation and recognition (Vahab et al. 2019).

\section{Literature Review}

As mentioned earlier about the various usages of object detection in business and other sectors as well, these usages are caused due to the need of various factors. In business sectors the usage of biometrics involves small process happening behind such as human behavioral detection namely facial recognition or iris code identification and finger-print detection and recognition to determine access allowed or denies for the authorized personnel working in the Company. Similarly, for security purposes the vehicles are being monitored by detecting the licensing plates as well as in health sector, the object detection has assisted in medical imaging making it more reliable. These required factors have led to the need for object detection and thereby its introduction being beneficial. Nowadays, even smart phones have facial recognition including other applications as well (Verschae and Ruiz-del-Solar 2015).

The implementation of real-time object detection one of which being pedestrian detection had made its way for a long time ever since the detectors such as ICF and HOG had introduced. The other long-established object detectors include the milestones and viola Jones. These detectors had their own features complying with the traditional technological enhancements.

However, since there was less technological aid, these detectors were insufficient in terms of its usefulness. The latest detection methods which is in the field of neural networks, namely FasterRCNN is an improved innovative approach as compared to the previously mentioned ones.

Apparently, the modern research and innovation of various such detection methods have increased to a vast area in facial recognition, pedestrian detection, text recognition, traffic light and signals detection, remote sensing detection of chosen object-interest, etc. These developments show the incremented efficiency in the object detection technology. Albeit these improvements, there are still drawbacks concerning the lack of multiple targets of object interest, inability to detect people where crowded due to differences in their behavioral factors and distinct features, challenges in identifying characters of a phrase or sentence, for instance, on sign boards, due to its size and other differences making it unreadable and difficulty in identifying and thereby recognize (Zou et al. 2019).

There are innumerable methods for object detection. But a real-time occupancy detection is quite less as compared to a general object detection technique. The latest types of such real-time occupancy detection are discussed in this section. The real-time object occupancy detection is a notion of detecting an object or a person being occupied in an area. However, if in an image the number of objects is individual then it can be maned as image localization, if there are more than one image then it is termed as object detection. Real-time object occupancy detection is useful in many situations, namely autonomous cars where the cars need not have a driver and therefore the vehicle may detect the objects in real-time such as pedestrians, other vehicles and the surrounding 
objects as well. There are many other useful circumstances for this concept as mentioned above, for face recognition, locating the object in an image along with detecting an action performed by the object. The usual algorithms for detecting objects are neural networks which has many versions which had mentioned previously.

When it comes to object occupancy, the foremost technique is Passive Infrared Sensors (PIR), the others include ultrasound, and image recognition. The PIR sensors determine object occupancy (mainly humans) by the change in heat produced by human from that of the surrounding. It can duly ascertain the rate of the person and locate the gap between sensor and human (Yun and Lee 2014). This technique is majorly used in automatic lighting system where a human presence is detected for switching on and off mechanism, also for other security purposes. This sensor is usually affordable and hence this can be utilized in both homes and organizations as well. PIR have been very useful during disaster to locate humans trapped underneath the debris, primarily during earthquakes (Biswa et al. 2013). Apart from PIR, the ultrasound, image recognition, acoustic also plays a key role in detecting object occupancy. The ultrasound detection works by estimating the differences in the frequency to evaluate a human occupant. The acoustic detector can locate an object using the resonance caused by the object (Harvey and OÝoung 2018). Thus, these detection techniques help recognizing objects in their own different ways.

\section{Human presence counting using PIR sensors}

There have been incidents reported where several times school children have slept off in buses and driver was unaware of the same and had the bus was shut down and stayed idle enclosed for a long period leading to the child's death. Similarly, an incident in an airplane where a woman had slept off. Such multiple incidents have occurred in the recent years. Albeit PIR sensors have utilized for object or person occupancy and motion detection, it is still underutilized for aspects such as these. This section is about discussion on PIR sensors for using it as a solution to the incidents mentioned above. PIR has always been a foremost motion detector prominently for identifying human presence by their movement and heat produced by them. There have been multiple studies held on this topic. The proposed idea is to be implemented in vehicles, specifically in transportation vehicles such as buses, airplanes.

The sensor shall recognize human presence whenever a person enters the vehicle as each of them will be counted and recorded in the system. And when they exit the vehicle the recorded count of passengers will be subtracted. Once the driver is certain about all passengers being dropped off the vehicle and when the vehicle is about to be turned off, the driver shall check the system whether the count of passengers is Zero and thus ensuring that the vehicle contain no more passengers, otherwise the system will automatically alert the driver after turning down the vehicle. This implementation idea not only ease the driver, it also benefits passengers who are unaware that they might have either slept in the vehicle or it may also occur where possibility of being in unconscious state, and thus assuring the safety of passengers as well. 


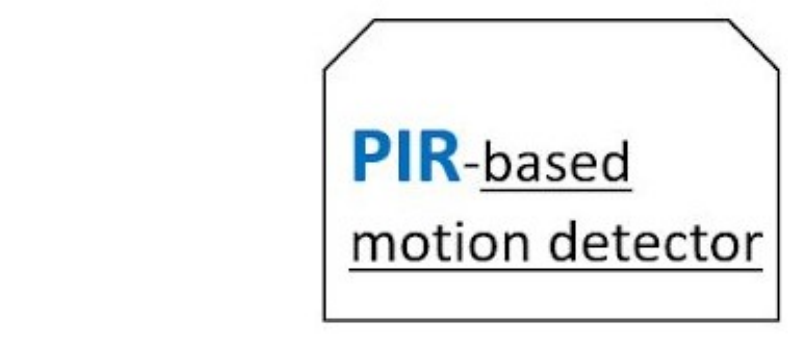

\section{Database}

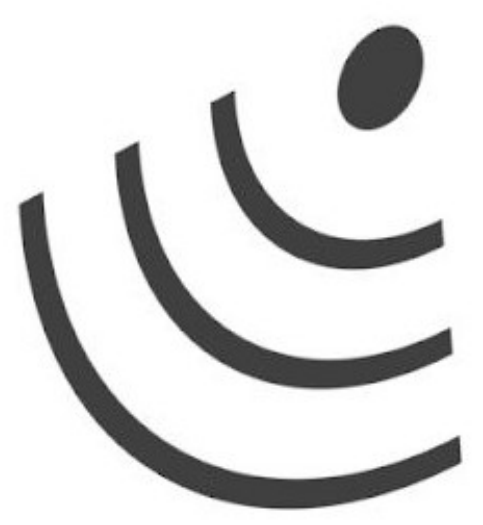

\section{Motion sensor detects person}

Records person count

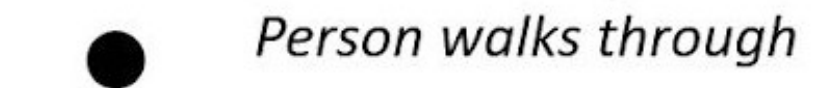

Figure 1. PIR detection and recording of person count.

The PIR is to be built-in the vehicle and has connection with a separate database to count and record the number of passengers that enters and decreases the count every time when a passenger exits the vehicle. The PIR-based motion detector can recognize a human either by their heat or by their movement. Also, the sensor is able to determine the gap between the person and the sensor itself.

\section{Utilizing SlimYOLOv3 for detecting objects in real- time}




\section{Journal of Student Research}

Fourth Middle East College Student Research Conference, Muscat, Sultanate of Oman

YOLO being categorized under RCNN, is most improved version of YOLO object detection technology. This section focuses on identifying objects in real-time scenarios for detecting lost objects by the passengers in the vehicle. People tend to lose objects that they carry while entering a vehicle and most of times this happens due to their negligence. Though there are many applications available to solve this issue by people themselves, it is still a difficult task to find what you lose in a transport vehicle. The idea is to implement SlimYOLOv3 framework for detecting object efficiently in real-time. The cameras embedded with YOLO object detection technology shall be installed in blind-spot region inside the vehicle, in simple words, the areas where human eye would find difficulty to view. For instance, if a passenger who had reported that any of their belonging objects was lost in the vehicle they had travelled before, using this advanced SlimYOLOv3 technology of YOLO the lost object can be easily found. 


\section{Journal of Student Research}

Fourth Middle East College Student Research Conference, Muscat, Sultanate of Oman
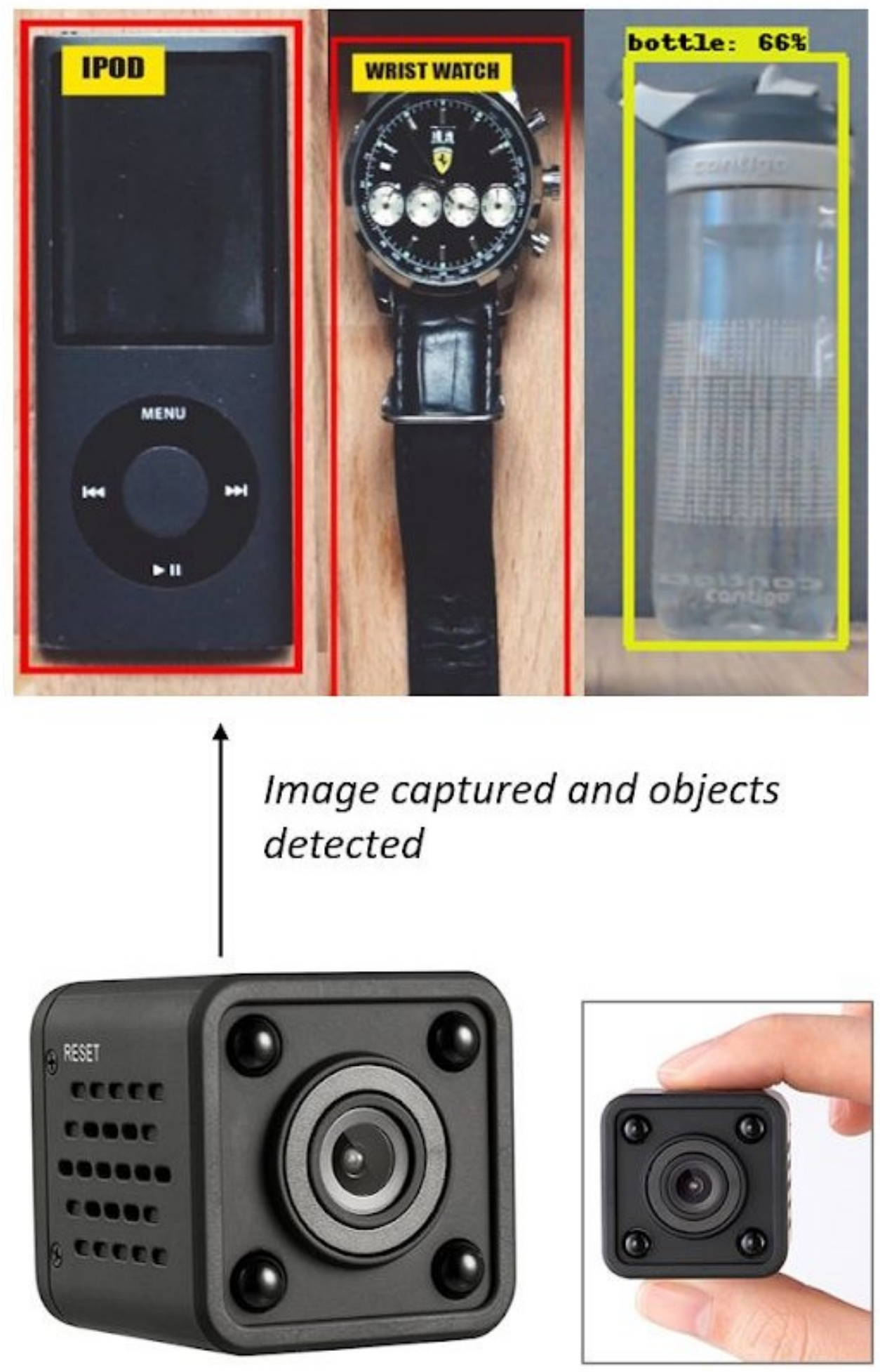

Figure 2. Objects detected using YOLO from image captured using motion sensing HD and night vision camera.

SlimYOLOv3 framework incorporated system and camera are installed which have features such as motion detection, with a 1080P HD, notify alerts and has a 64G infrared night vision, which is perfect in terms of capturing images at any time whether it be during day or night. Using YOLO, 
lost objects can be found from blind spots by setting up the compatible camera as shown in the figure above, through which image captured can be recognized and retrieved. The usage of this feature shall be used inside transport vehicles where higher possibility for losing one's belonging is present and finding them are sometimes difficult.

\section{Future Enhancements}

As described so far about both the PIR detector and SlimYOLOv3 object detector, their efficiency is proven and consists of perks to be utilized and incorporated technically in situations where keeping passengers safe as well as providing assist in finding the passenger's belongings once lost. Both these techniques are currently specified as functioning on separate managing platform. However, it will be a great innovation step for converging both these concepts to be embedded together as one robust system so to produce the most technical efficiency by utilizing them simultaneously.

\section{Conclusion}

There have been numerous studies done based on object detection as well as occupancy of human in the field of YOLO categorized under RCNN and in PIR-based motion detectors to do automatic functions such as lighting, music, escalators, automatic doors operations which created another evolution in automation. Nevertheless, this aspect has not been effectively seen its way through passenger transportation sector. Joining PIR technology and YOLO framework emerges a deep learning in the Computer Vision field. Pacing along with these ideas and implementing it in the same concept of automation is an advancement leading towards achieving true future technology aspect. Acknowledging the reality of the mentioned incidents in the paper which have brought less or absolutely no solution until now, brought the intention in doing the research. Implementing the technology detailed in the research paper has the potential to deliver what it is termed to achieve. This notion is believed to see through as an eye-opening solution to reduce the occurrence of such incidents.

\section{References}

Raykov, Y., et al. (2016). "Predicting room occupancy with a single passive infrared (PIR) sensor through behavior extraction." The 2016 ACM International Joint Conference, pp. 1016-1027.

Hirano, Y., Garcia, C., Sukthankar, R., Hoogs, A. (2006). "Industry and Object Recognition: Applications, Applied Research and Challenges." Conference Paper, pp. 49-64.

Zou, Z., et al. (2019). “Object Detection in 20 Years: A Survey.” IEEE. 1-31.

Redmon, J., Divvala, S., Girshick, R., Farhadi, A. (2016). "You Only Look Once: Unified, Real-Time Object Detection." University of Washington., 1- 10.

Srinivasan, P., et al. (2014). "Locate Misplaced Objects! GPS-GSM-Bluetooth Enabled Tracking” International Journal of Computer Trends and Technology (IJCTT)., 9(1): 1-10.

Rizaev, I., et al. (2018). "Method of Automatic Detection of New Motionless Objects on the Basis of Comparison of Images of the Area." 2018 3rd Russian-Pacific Conference on Computer Technology and Applications (RPC) 1-6.

Vahab, A., et al. (2019). “Applications of Object Detection System .” International Research Journal of Engineering and Technology (IRJET), 6(4): 4186-4192.

Raghav, P., et al. (2004). “Object Detection Algorithms for Video Surveillance Applications.” 2018 


\section{Journal of Student Research}

Fourth Middle East College Student Research Conference, Muscat, Sultanate of Oman

International Conference on Communication and Signal Processing (ICCSP), pp. 0563-0568.

Kwon, J., et al. (2018). “Object Bounding Box-Critic Networks for Occlusion-Robust Object Detection in Road Scene." 2018 25th IEEE International Conference on Image Processing (ICIP)., pp. 1313-1317.

Kamnoonwatana,N., et al. (2014). "Unattended and stolen object detection based on relocating of existing object.” 2014 Third ICT International Student Project Conference (ICT-ISPC)., pp. 115-118.

Harvey, B., O’Young, S. (2018). “Acoustic Detection of a Fixed-Wing UAV.” Drones 2018, pp. 1-18.

Yun, J., Lee, S. (2014). "Human Movement Detection and Identification Using Pyroelectric Infrared Sensors." Biomedical Sensors and systems. 14 (5): 8057-8081.

Ruiz-del-Solar, J., Verschae, R. (2015). “Object Detection: Current and Future Directions.” Front. Robot. AI 2015.

Wang, Y., et al. (2018). “Occupancy Detection and Localization by Monitoring Nonlinear Energy Flow of a Shuttered Passive Infrared Sensor" IEEE Sensors Journal., pp. 1-1.

Pawar, Y., et al. (2018). "Motion Detection Using PIR Sensor." International Research Journal of Engineering and Technology (IRJET), 5(4): 4753-4756.

Ren, S., et al. (2015). "Faster R-CNN: Towards Real-Time Object Detection with Region Proposal Networks." Microsoft Research, pp. 1-9. 